\title{
Optimal Destabilization of DNA Double Strands by Single- Nucleobase Caging
}

\author{
Patrick Seyfried $^{+}{ }^{[a]}$ Marcel Heinz $^{+}{ }^{[b]}$ György Pintér ${ }^{+},{ }^{[c]}$ Dean-Paulos Klötzner, ${ }^{[a]}$ \\ Yvonne Becker, ${ }^{[a]}$ Michael Bolte, ${ }^{[d]}$ Hendrik R. A. Jonker, ${ }^{[c]}$ Lukas S. Stelzl, ${ }^{[b]}$ \\ Gerhard Hummer, ${ }^{*[b, e]}$ Harald Schwalbe, ${ }^{*[c]}$ and Alexander Heckel ${ }^{*[a]}$
}

\begin{abstract}
Photolabile protecting groups are widely used to trigger oligonucleotide activity. The ON/OFF-amplitude is a critical parameter. An experimental setup has been developed to identify protecting group derivatives with superior caging properties. Bulky rests are attached to the cage moiety via Cu-catalyzed azide-alkyne cycloaddition post-synthetically on DNA. Interestingly, the decrease in melting temperature upon introducing o-nitrobenzyl-caged (NPBY-) and diethylaminocoumarin-cages (DEACM-) in DNA duplexes reaches a limiting value. NMR spectroscopy was used to
\end{abstract}

characterize individual base-pair stabilities and determine experimental structures of a selected number of photocaged DNA molecules. The experimental structures agree well with structures predicted by MD simulations. Combined, the structural data indicate that once a sterically demanding group is added to generate a tri-substituted carbon, the sterically less demanding cage moiety points towards the neighboring nucleoside and the bulkier substituents remain in the major groove.

\section{Introduction}

Photolabile protecting groups have become a powerful tool in biochemical and biomedical research. ${ }^{[1-5]}$ "(Photo)caged" targets such as neurotransmitters, ${ }^{[6,7]}$ peptides, ${ }^{[8]}$ proteins, ${ }^{[9-11]}$ me-

[a] Dr. P. Seyfried, Dr. D.-P. Klötzner, Y. Becker, Prof. Dr. A. Heckel Institute for Organic Chemistry and Chemical Biology Goethe University Frankfurt

Max-von-Laue-Str. 7, 60438 Frankfurt am Main (Germany)

E-mail: heckel@uni-frankfurt.de

Homepage: www.photochem.uni-frankfurt.de

[b] M. Heinz, ${ }^{+}$Dr. L. S. Stelzl, Prof. Dr. G. Hummer Department of Theoretical Biophysics

Max Planck Institute of Biophysics

Max-von-Laue-Str. 3, 60438 Frankfurt am Main (Germany)

E-mail: gerhard.hummer@biophys.mpg.de

Homepage: www.biophys.mpg.de/en/theoretical-biophysics.html

[c] G. Pintér, ${ }^{+}$Dr. H. R. A. Jonker, Prof. Dr. H. Schwalbe

Institute for Organic Chemistry and Chemical Biology

Goethe University Frankfurt/

Centre for Biomolecular Magnetic Resonance (BMRZ)

Max-von-Laue-Str. 7, 60438 Frankfurt am Main (Germany)

E-mail:schwalbe@nmr.uni-frankfurt.de

Homepage: www.schwalbe.org.chemie.uni-frankfurt.de

[d] Dr. M. Bolte

Institute for Inorganic Chemistry, Goethe University Frankfurt

Max-von-Laue-Str. 7, 60438 Frankfurt am Main (Germany)

[e] Prof. Dr. G. Hummer

Institute of Biophysics

Max-von-Laue-Str. 1, 60438 Frankfurt am Main (Germany)

$\left.{ }^{[+}\right]$These authors contributed equally to this work.

$\square$ Supporting information and the ORCID identification number(s) for the au-

(iD thor(s) of this article can be found under:

https://doi.org/10.1002/chem.201804040. tabolites, $^{[12]}$ antibiotics $^{[13]}$ and oligonucleotides ${ }^{[14-16]}$ can be trapped in an inactive state. Upon irradiation with light, the protecting group is removed, releasing the active target with high spatial and temporal control.

A wide scope of in vitro and in vivo applications has been reported for caged oligonucleotides. Antisense strategies, ${ }^{[17]}$ siRNA, ${ }^{[18,19]}$ miRNA $^{[20]}$ and aptamers ${ }^{[21]}$ have been employed successfully for the photo-regulation of gene expression and protein interaction. Caged oligonucleotides have also been used in transcriptomics, ${ }^{[22,23]}$ studies of RNA and DNA folding $^{[12,24,25]}$ RNA-protein interactions ${ }^{[17,26]}$ and RNA therapeutics ${ }^{[27,28]}$ exist. Different caging strategies for oligonucleotides have been developed. The photolabile group can either be attached to the nucleobases at the Watson-Crick or the major groove site, at the phosphate backbone or the sugar moiety. Caging of the Watson-Crick site perturbs the base pairing and destabilizes the duplex. However, complete duplex destabilization and hence high ON/OFF-amplitude is often not achieved without incorporating multiple protecting groups at different positions in the sequence. As a consequence, long irradiation times and high doses of light are required to trigger a change, which limits biophysical and biological applications.

Different approaches have been reported to overcome this challenge. One of them is to link a partially complementary inhibitor strand to the oligonucleotide, which is cleaved and removed upon irradiation. ${ }^{[29]}$ Another one is the cyclization of functional oligonucleotides with a photolabile linker. ${ }^{[30-32]}$ While the former requires optimization of the inhibitor strand length, the latter is dependent on the ring size.

Increasing the steric demand of photolabile protecting groups is another promising approach, as shown for the 
caging of proteins ${ }^{[33]}$ and plasmids. ${ }^{[34]}$ Ellis-Davies et al. recently reported cloaking of caged GABA with dendrimers via click chemistry. ${ }^{[35]}$ Particularly with regard to in vivo applications, there is, however, still a considerable need for optimized oligonucleotide caging strategies. In this contribution, we investigate and maximize the destabilization of duplexes by a single photolabile protecting group.

In our approach, photolabile protecting groups are attached at the Watson-Crick site of nucleobases. We expected that an additional bulky residue directly connected in $\alpha$-position of the cage moiety would increase the duplex destabilizing effect by introducing more steric repulsion (Figure $1 \mathrm{a}$ ). Further, the bulk-

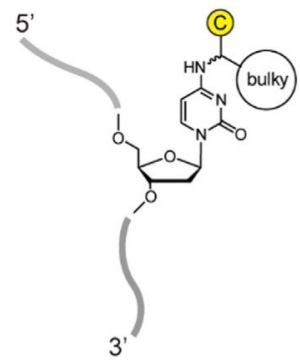

b

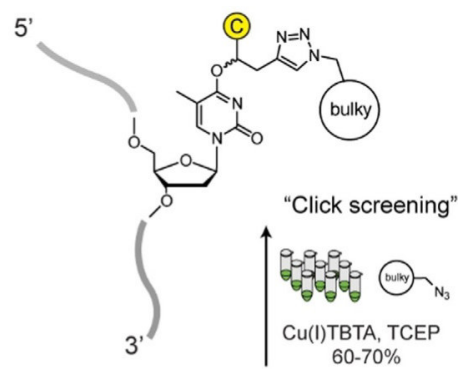

sequence 1: 5'-GCA TAT AXT AAG GTG-3' sequence 2: 5'-GCA TAAAXAAAG GTG-3'

$$
\text { (C) = } \mathrm{Et}_{2} \mathrm{~N} \text { or } \mathrm{O}
$$

Figure 1. Duplex stability of photocaged DNA is decreased by adding bulky residues at the cage moieties, increasing steric complexity. Bulky residues are attached (a) directly in $\alpha$-position or (b) via Cu-catalyzed azide-alkyne cycloaddition (CuAAC) with the alkyne-modified nitrobenzyl-(NPBY) and coumarinyl-(DEACBY) caged nucleobases.

iness of the substituents at the $\alpha$-position can be increased by introducing a propargyl functional group, which can react with bulky azide-residues after the solid-phase synthesis of DNA. Employing Cu-catalyzed azide-alkyne cycloaddition (CuAAC), we screened the impact of a number of bulky residues on duplex stability (Figure $1 \mathrm{~b}$ ). To rationalize the experimentally determined effects on melting temperatures, we characterized individual base-pair stability around the nucleotide derivatized with the photocage by NMR spectroscopy. Also, we determined experimentally the impact of the configuration on duplex stability and developed NMR-based structures of the photocaged DNA for some members of the series. In order to provide a rapid method to determine structures of the series of 20 differently photocaged DNAs, we also employed molecular dynamics (MD) simulations and established that very similar structures are determined by NMR and MD. Building on continuous improvement of force fields, ${ }^{[36-40]}$ we were able to use MD simulations to elucidate, at atomic resolution, the interactions between the novel nucleobases and the DNA double helix. $^{[41,42]}$

\section{Results and Discussion}

\section{Impact of steric bulkiness and stereochemistry on duplex stability}

Following a previous study by Steinert et al., which showed the impact of the absolute configuration of the 1-(2-nitrophenyl)ethyl (NPE) photocage on the local and global DNA duplex stability, ${ }^{[43]}$ we started our investigations with a nitro-phenyl type photocage (2-nitrophenyl)(phenyl)methyl (NPPM) (Figure 2). In this design, the methyl group at the $\alpha$-position of the previous study was replaced by a sterically more demanding phenyl group. Both enantiomers of the NPPM cage were synthesized and attached to 2'-deoxycytidine. The diastereomeric phosphoramidites were used for the DNA solid-phase synthesis of NPPM-photocaged 15-mer DNA (sequence 1, see Figure 1).

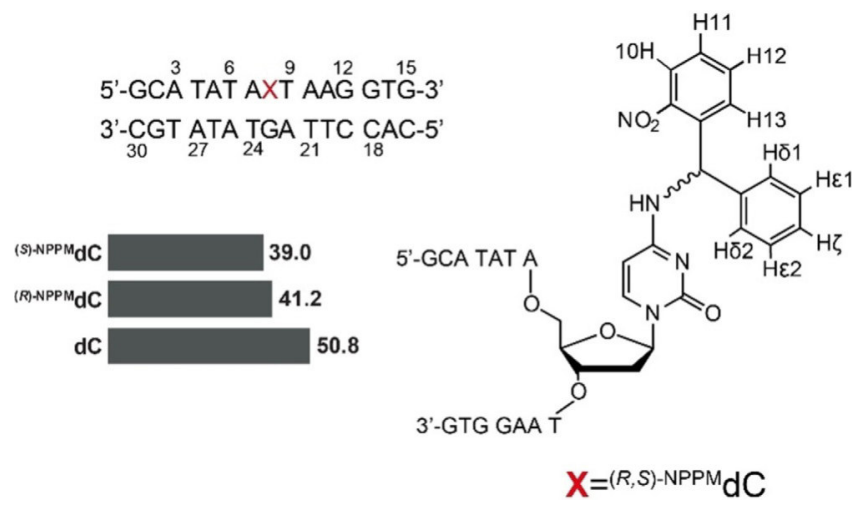

Figure 2. Initial series of NPPM-photocaged DNA and the respective melting points of the native, (R) and (S)-NPPM caged DNA, including the numbering scheme used in this study.

To investigate the effect of the bulky phenyl group on the DNA duplex stability, we first determined their melting temperature. Compared to the native DNA duplex, the (R)-NPPM- and the (S)-NPPM-cages lower the melting point by $9.6^{\circ} \mathrm{C}$ and $11.8^{\circ} \mathrm{C}$, respectively. Indeed, the bulky phenyl residue exceeds the destabilizing effect of a methyl group: compared to the NPE-caged DNA duplex used previously we observed additional decrease of melting temperatures by $5.6^{\circ} \mathrm{C}$ for the $(R)$ - and $2.2^{\circ} \mathrm{C}$ for the (S)-configuration.

Interestingly, the melting points of the NPPM enantiomers are closer than those of the NPE enantiomers. This comparison suggests that the increasing size of the photolabile group induces further destabilization in the DNA duplex and the small difference between the diastereomeric photocaged DNA duplexes might be a result of the very similar size of the nitrophenyl and phenyl moiety.

\section{Determination of the absolute configuration of NPPM-modi- fied DNA}

In order to determine the absolute configuration of the two diastereomeric DNA strands that differ in the configuration at 
the $\alpha$-position of the NPPM cage moiety, we analyzed the NOESY spectra of the separated diastereomers, which indeed showed a different set of peaks around the incorporation site as expected (Figure 3). The NMR signals stemming from the photocage moiety were assigned using ${ }^{1} \mathrm{H},{ }^{1} \mathrm{H}$ NOESY and ${ }^{1} \mathrm{H},{ }^{1} \mathrm{H}$ TOCSY spectra. The nitro-phenyl group signals show high dispersion in the less crowded aromatic region. Hence, based on a model structure derived from our previous study ${ }_{1}^{[43]}$ we could clearly distinguish between $(S)$ - and $(R)$-configuration.
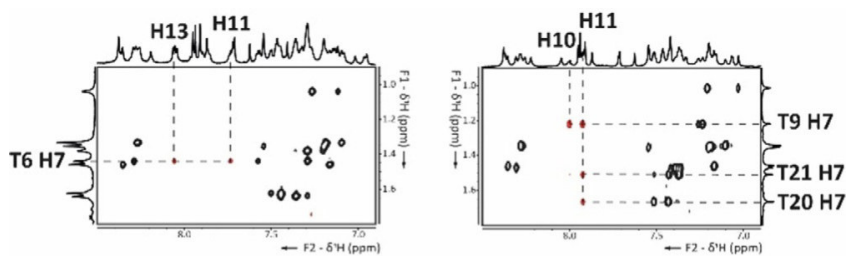

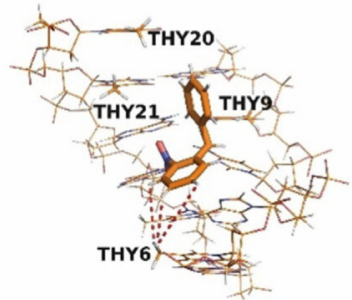

S-configuration

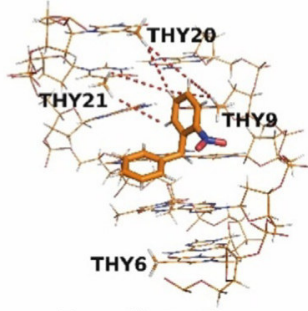

$R$-configuration
Figure 3. Upper part: NOESY spectra of aromatic (6.9-8.5 ppm) methyl (0.9$1.8 \mathrm{ppm}$ ) region. Peaks highlighted in red are correlations between the nitro-phenyl signals of the photocage and the signals of the thymidine methyl group; numbering scheme is according to Figure 2. Lower part: 3D model of the photocaged DNA duplex derived from our previous studies ${ }^{[43]}$ showing the middle part of the duplex-highlighting the neighboring methyl groups. Red dashed lines show correlations observed in NOESY spectra.

\section{Structure determination of ( $R$ )- and (S)-NPPM caged DNA duplex and structural basis of decreased stability}

To analyze the structural basis of the reduced stability and the differences in melting point depression for the different diastereomers, we determined the structure around the modified DNA base pairs using ${ }^{1} \mathrm{H},{ }^{1} \mathrm{H}$ NOESY restraints. Exchangeable proton signals were assigned from ${ }^{1} \mathrm{H},{ }^{1} \mathrm{H}$ NOESY spectra recorded in $\mathrm{H}_{2} \mathrm{O} / \mathrm{D}_{2} \mathrm{O}$ (9:1) sample. Non-exchangeable proton signals except for $\mathrm{H}^{\prime}$ and $\mathrm{H}^{\prime \prime}$ " were assigned based on the sequential walk between $\mathrm{H}^{\prime}{ }^{\prime}-\mathrm{H} 6 / 8$ and $\mathrm{H}^{\prime} / \mathrm{H} 2^{\prime \prime}-\mathrm{H} 6 / \mathrm{H} 8$ from ${ }^{1} \mathrm{H},{ }^{1} \mathrm{H}$ TOCSY, ${ }^{1} \mathrm{H},{ }^{1} \mathrm{H}$ NOESY recorded in a $\mathrm{D}_{2} \mathrm{O}$ sample. All of the imino protons were observable indicating that a single photocage does not fully disrupt the base pair formation at low temperature (288 K). CD spectra of the caged DNA (see the Supporting Information) showed typical B-helix secondary structure supporting that the duplex secondary structure is stable. Compared to the native DNA, only the signals of T6, T9, T24 and G23 around the site of modification show significant chemical shift changes.

With nearly $80 \%$ of the proton signals assigned, structure calculations were carried out using the ARIA/CNS software package with spin diffusion correction to be able to incorporate NOE peaks from longer mixing times, following our previ- ously established protocol (Steinert et al.). ${ }^{[43]}$ In both isomers, the aromatic moiety of the photocage is positioned within the major groove (Figure 4).

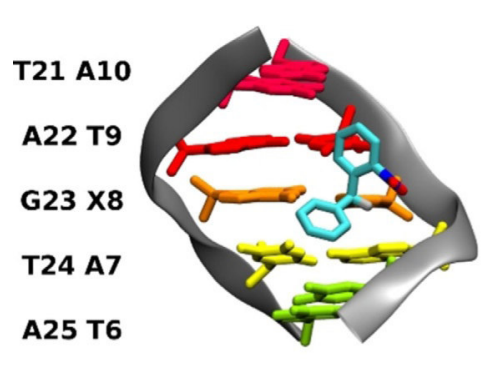

(R)NPPM dC NOESY Structure

T21 A10

A22 T9

G23 X8

T24 A7

A25 T6

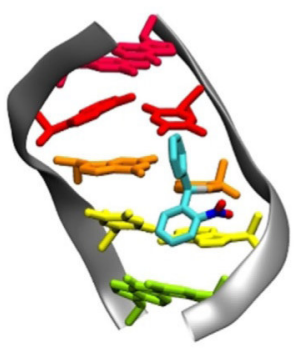

(S)NPPM dC

NOESY Structure

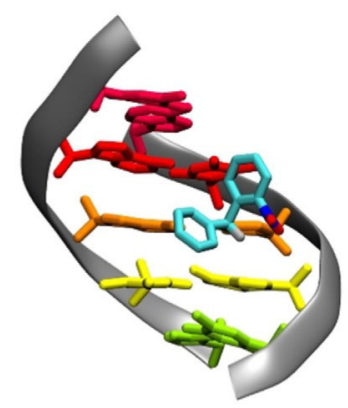

(R)NPPM dC MD Cluster 1

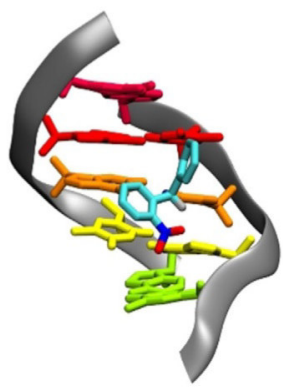

(S)NPPM dC

MD Cluster 1
Figure 4. Central five base pairs of the NOESY-based structures of $(R)$ - and (S)-NPPM-caged DNA and the representative structures of the highest populated RMSD cluster in MD simulations of the same system. The NOESY-based structures (left) have the lowest energy in the structure calculation. The RMSD of the inner three base pairs compared to mean structure of the final structure bundle is $0.70 \AA$ and $0.91 \AA$ for $(R)$ - and (S)-NPPM-caged DNA, respectively. The MD structures (right) represent the centers of cluster 1 , as obtained by clustering on the central base pairs with an RMSD cutoff of $1.25 \AA$.

At low temperature $(288 \mathrm{~K})$, the cross-strand hydrogen bond base pair is not fully disrupted, as evidenced by observed G23 imino proton (Figure 5). The linewidth of the imino proton is, however, larger compared to the unmodified signal in line with decreased hydrogen bond strength. Measurement of imino proton exchange rates (see Supporting Information) also show increased exchange rates with water, in line with more frequent opening of the base pair and concomitant destabili-

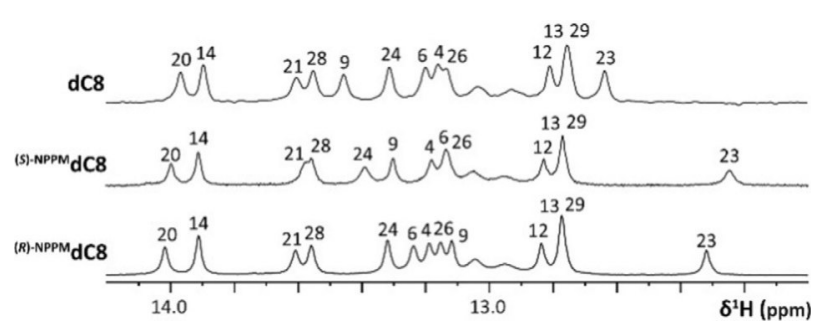

Figure 5. Imino proton region of $1 \mathrm{H}$ NMR spectra $(600 \mathrm{MHz})$ of unmodified (top), (S)-NPPM- (middle) and (R)-NPPM- (bottom) caged DNA duplex. For numbering scheme, see Figure 2. 
zation of the hydrogen bond. Furthermore, in both $3^{\prime}$ - and 5'direction the neighboring two base pairs are less stable compared to the unmodified DNA.

There are no major differences in the structure of the two isomers (Figure 4). Apparently, the similar size of the phenyl moiety and the nitro-phenyl group and the requirement to fit the alternative groups in the major groove lead to the small $\Delta \Delta T_{\mathrm{m}}$ of $2.2^{\circ} \mathrm{C}$ between the two isomers (see also the comment in the last paragraph two sections above). From the structure, we speculate that the reduced stability originates from the attached cage inducing tension in the backbone to accommodate the modified base in the double helix, as the nucleobase does not flip out to the solvent. This is most likely energetically favored due to the $\pi-\pi$ stacking of nucleobases and the hydrogen bond formation. Although the backbone still adopts a B-form conformation, the position of the nucleobases around the modified nucleotide deviate from the optimal position, reducing the stabilizing $\pi-\pi$ stacking effect.

Further, we performed molecular dynamics (MD) simulations of the $(R)$ - and (S)-NPPM caged dsDNA (see Supporting Information). From twenty independent simulations, with a cumulative simulation time of $2 \mu$ s per cage, we calculated the distances for which NOE contacts between cage and the DNA had been measured. In a comparison of calculated and NOE-inferred distances, we obtained quantitative agreement matching 76 out of 80 experimental NOEs for (R)-NPPM, and 48 out of 51 for (S)-NPPM, between MD simulations and experiment. To characterize the sampled conformation space, we clustered the MD structures according to their central base pairs with a $1.25 \AA$ RMSD cut-off (see Supporting Information) and representative structures of the highest populated cluster are shown in Figure 4. We observed free rotation of the NPPM phenyl group, which agrees with the experimental observation of identical chemical shifts for $\mathrm{H} \delta$ and $\mathrm{H} \varepsilon$. This comparison shows the predictive power of MD simulations for caged DNAs. On this basis, we use MD simulations to understand the caging properties, when no NMR structures are available.

To check for the generality of the previous statements regarding the influence of steric demand on the $\alpha$-position of an NPE photocage we replaced the phenyl moiety in the NPPM group with an adamantyl group and installed it on T9 in sequence 1 (Figure 1). We will refer to this cage as NPAM for (2nitrophenyl)(adamantyl)methyl. The two diastereomeric DNA sequences were separated by RP-HPLC. While the melting point of the NPAM-photocaged DNA could be further decreased compared to the NPPM-photocaged DNA $\left(\Delta T_{\mathrm{m}}=12.2 /\right.$ $10.7^{\circ} \mathrm{C}$ compared to $11.8 / 9.6^{\circ} \mathrm{C}$ ), the $\Delta \Delta T_{\mathrm{m}}$ difference between the diastereomers amounts to a minimal value of $1.5^{\circ} \mathrm{C}$. This supports the hypothesis that with additional steric demand at this position the destabilizing effect levels off.

\section{Screening of the duplex stability of bulky caged DNA with a post-synthetic CuAAC click reaction}

Having characterized a DNA duplex containing the very bulky NPPM- and the NPAM-photocage groups attached to the $\alpha$-position of the cage moiety following this stepwise synthetic ap- proach, we extended our strategy in order to rapidly screen several modifying groups with different steric requirements. We used an almost identical 15-mer model DNA (Sequence 2, Figure 1$)^{[44,45]}$ and incorporated, using standard DNA solidphase synthesis, either 1-(2-nitrophenyl)but-3-yn- (NPBY) or 7(diethylamino)-4-(but-3-yn-1-yl)coumarin (DEACBY) caged dT at the center position (Figure 6). The synthesis of both $\mathrm{dT}^{\mathrm{NPBY}}$ and $\mathrm{dT}^{\mathrm{DEACBY}}$ phosphoramidites has been reported in a recent publication. ${ }^{[30]}$ A library of azido compounds was generated from

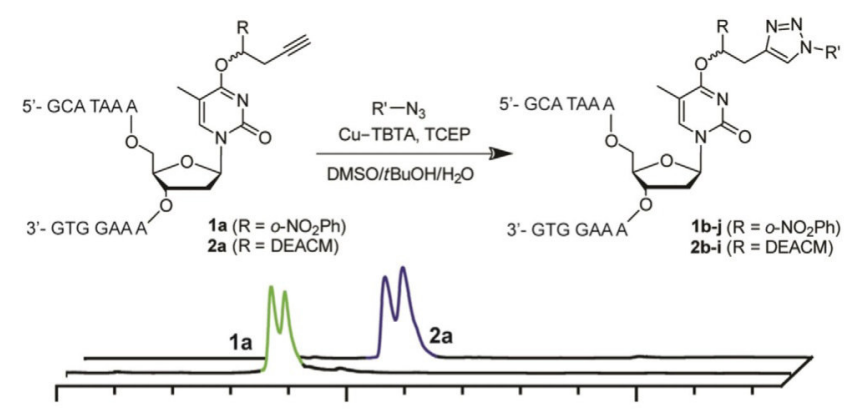

$\mathrm{R}^{\prime}=$

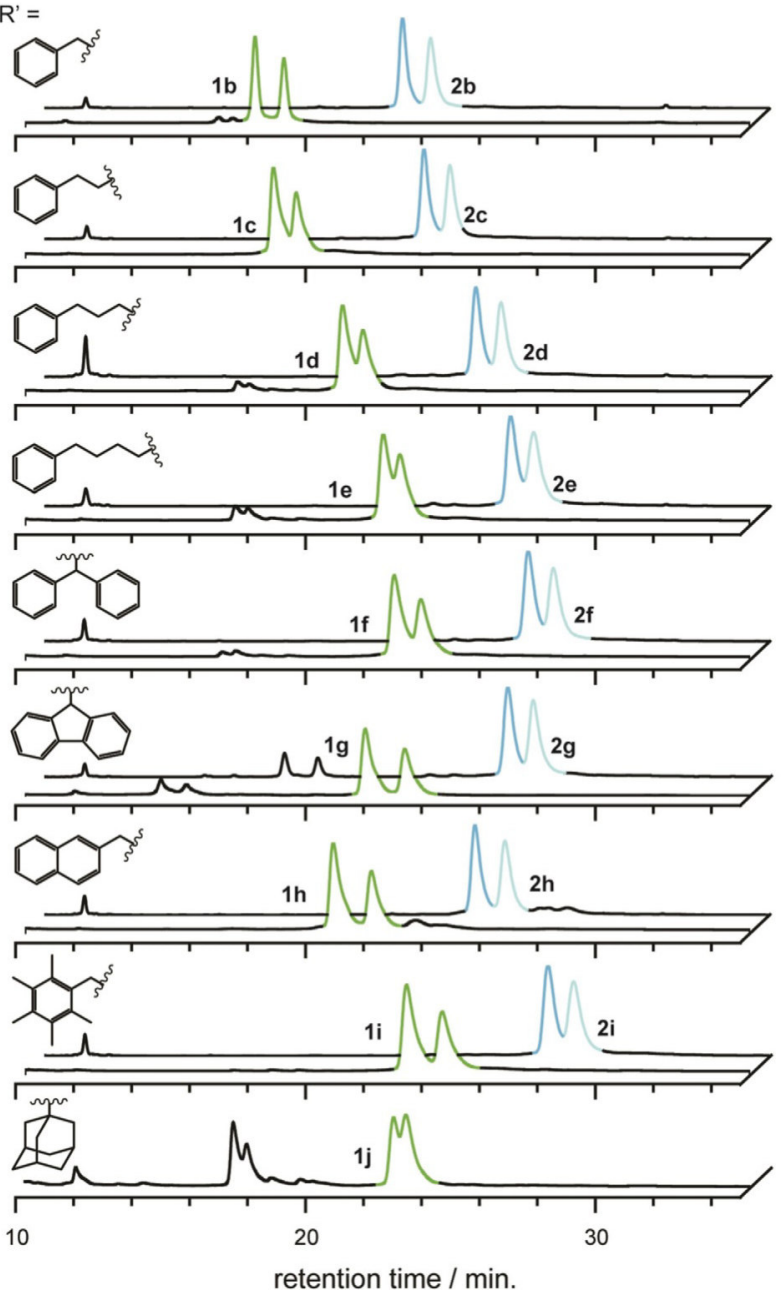

Figure 6. RP-HPLC monitoring of the CUAAC reaction after $18 \mathrm{~h}$. Alkynemodified caged DNA $\mathbf{1}$ a or $\mathbf{2}$ a was conjugated with different bulky triazole residues to afford the diastereomers $\mathbf{1} \mathbf{b}-\mathbf{j}$ and $\mathbf{2} \mathbf{b}-\mathbf{i}$. Diastereomeric product peaks in the ortho-nitrobenzyl series and in the coumarin series are colored in green and blue, respectively. 
the bromide precursors by simple nucleophilic substitution (for details see Table S1 in the Supporting Information). Substituents with varying degrees of bulkiness were introduced by a post-synthetic CUAAC click reaction with a 1:10 DNA/azide ratio using $\mathrm{CuSO}_{4} / \mathrm{TBTA}$ and TCEP in a solvent mixture of water/DMSO/tBuOH buffered with $0.1 \mathrm{M}$ TEAA (Figure 6). In general, the potentially limited accessibility of the alkyne represented no problem in the CuAAC click reaction.

Products were obtained after RP-HPLC purification as diastereomeric mixture typically in $60-70 \%$ yields. No reaction was observed with azidoadamantane at room temperature. Thus, we repeated the reaction with microwave assistance at $60^{\circ} \mathrm{C}$, where reasonable product conversion was achieved within $1 \mathrm{~h}$. Release of the protecting group was also observed in small amounts.

We tested the duplex destabilization effect in this second series of DNA oligonucleotides. We first investigated the melting temperatures of the DNA derivatives $1 \mathbf{a}-\mathbf{j}$ (NPBY-screening) and the derivatives $\mathbf{2} \mathbf{a}-\mathbf{i}$ (DEACBY-screening) as diastereomeric mixtures. The results are summarized in Figure 7 (black

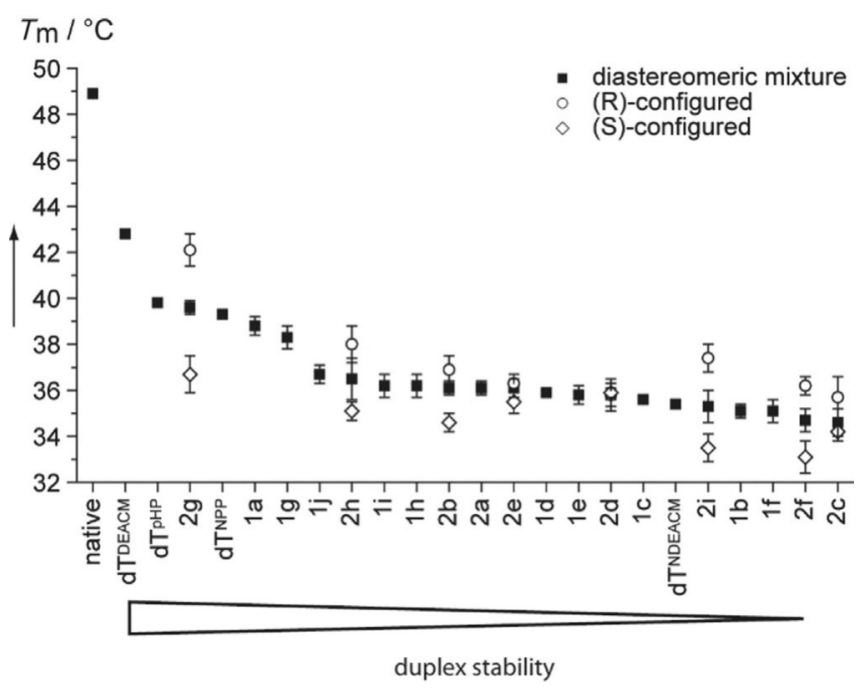

Figure 7. Melting temperature studies of caged model dsDNA (15-mer) $\mathbf{1} \mathbf{a}-\mathbf{j}$ and $\mathbf{2} \mathbf{a}-\mathbf{i}$ after attachment of bulky substituents by CuAAC click reaction ordered by decreasing $T_{\mathrm{m}}$ values of the diastereomeric mixtures. For DEACBYcaged derivatives $\mathbf{2} \mathbf{b}$-i $(S)$ - and (R)-DEACBY-caged are analyzed additionally.

squares) and a full overview is given in Figure S9 (Supporting Information). Prior to the click reaction, the incorporation of the parent NPBY-protecting group (1 a) led to a decrease of melting temperature from $48.9^{\circ} \mathrm{C}$ (native duplex) to $38.8^{\circ} \mathrm{C}$. This value is above or in the same range as observed for most of our previously investigated protecting groups. Previously obtained values with 7-diethylaminocoumarin-4-yl)methyl (DEACM), $p$-hydroxyphenacyl ( $p \mathrm{HP}$ ) and 2-(o-nitrophenyl)propyl (NPP) caging groups are included for reference purposes in Figure 7.

After click conjugation, all bulky NPBY-caged oligonucleotides $\mathbf{1} \mathbf{b}$-j exhibited additional duplex destabilization. The lowest melting temperature of $35.1^{\circ} \mathrm{C}$ was observed for the benzyl- and the diphenyl-triazole residues $\mathbf{1} \mathbf{b}$ and $\mathbf{1} \mathbf{f}$ in the
NPBY-screening. Surprisingly, the adamantyl-(1 $\mathbf{j})$ and the fluorenyl-triazole $\mathbf{1} \mathbf{g}$ give only a little additional effect. Altogether, the bulky modifications here extend the effect of a single NPBY- protecting group by 0.5 to $3.7^{\circ} \mathrm{C}$. With a melting temperature of $37.6{ }^{\circ} \mathrm{C}$, the parent $D E A C B Y$ derivative $2 \mathrm{a}$ decreased the duplex stability slightly more than the parent NPBY derivative $1 \mathrm{a}$. This observation might result from the larger size of the coumarin moiety. Bulky residues at the triazole-site again caused an additional decrease of measured melting temperatures in the screening of oligonucleotides $\mathbf{2} \mathbf{b}$ i. In case of phenylethyl-triazole $\mathbf{2 c}$, we observed the lowest overall value of $34.6{ }^{\circ} \mathrm{C}$, which is even $0.5^{\circ} \mathrm{C}$ lower compared to the lowest NPBY-derivatives. Relative to the parent DEACBY derivative $\mathbf{2} \mathbf{a}$, the fluorenyl-triazole $\mathbf{2} \mathbf{g}$ stabilized the DNA duplex by $2^{\circ} \mathrm{C}$. Altogether, our first screening clearly identified two derivatization patterns with eminent destabilization properties, namely the phenylethyl- and the diphenyl-triazoles $1 \mathrm{c} /$ $\mathbf{2 c}$ and $\mathbf{1} \mathbf{f} / \mathbf{2} \mathbf{f}$ (Figure 7).

\section{Influence of the absolute configuration on caged DNA duplex stability and uncaging efficiency}

Also in the second series of optimization of the photocaging groups, we investigated the effect of the absolute configuration of the $\alpha$-position of the cage moiety on duplex stability. ${ }^{[43]}$ For these experiments, we focused on DEACBY-DNA derivatives $\mathbf{2} \mathbf{b}-\mathbf{i}$, since in contrast to NPBY, the DEACBY protecting group can be removed efficiently using wavelengths above $400 \mathrm{~nm}$, which make these compounds great candidates for biological applications. Of each derivative $\mathbf{2} \mathbf{b}-\mathbf{i}$, the respective diastereomers were separated by RP-HPLC. In order to assign the absolute configurations based on RP-HPLC retention properties, we required an HPLC-reference with known configuration and hence enantioselectively synthesized the DEACBY-protecting group.

Previously, asymmetric propargylation of aldehydes was reported using the chiral Brønsted acid catalyst $(R)$-TRIP-PA. ${ }^{[46,47]}$ In these studies, high enantiomer ratios were obtained with non-polar solvents like cyclohexane or toluene. Since the coumarin aldehyde 3 shows poor solubility in those solvents, we optimized the procedure by using a solvent mixture of cyclohexane and dichloromethane $(4: 1)$, while keeping the temperature at $10^{\circ} \mathrm{C}$. Under these conditions, we observed almost full conversion within $48 \mathrm{~h}$ reaching $88 \%$ ee (Scheme $1 \mathrm{a}$ ). The main product of the reaction in presence of $(R)$-TRIP-PA was expected to be the $(R)$ configuration, which was confirmed by Mosher ester analysis and X-ray crystal structure analysis of the Mosher derivative of (S)-4 (Figure S2 in the Supporting Information). (R)-4 was attached to triazole-activated thymidine 6 (Scheme $1 \mathrm{~b}$ ).

After cleavage of the TBDMS groups with TBAF and coupling of the DMTr protecting group to the $5^{\prime}-\mathrm{O}$ position it was converted into the phosphoramidite building block 10 with an overall yield of $40 \%$. This building block was then used to prepare at first DEACBY parent DNA $(R)-\mathbf{2}$ a and after click reaction also the bulky derivatives $(R)-\mathbf{2} \mathbf{b}-\mathbf{i}$. Performing co-injection experiments on RP- HPLC we could then confirm that the early 

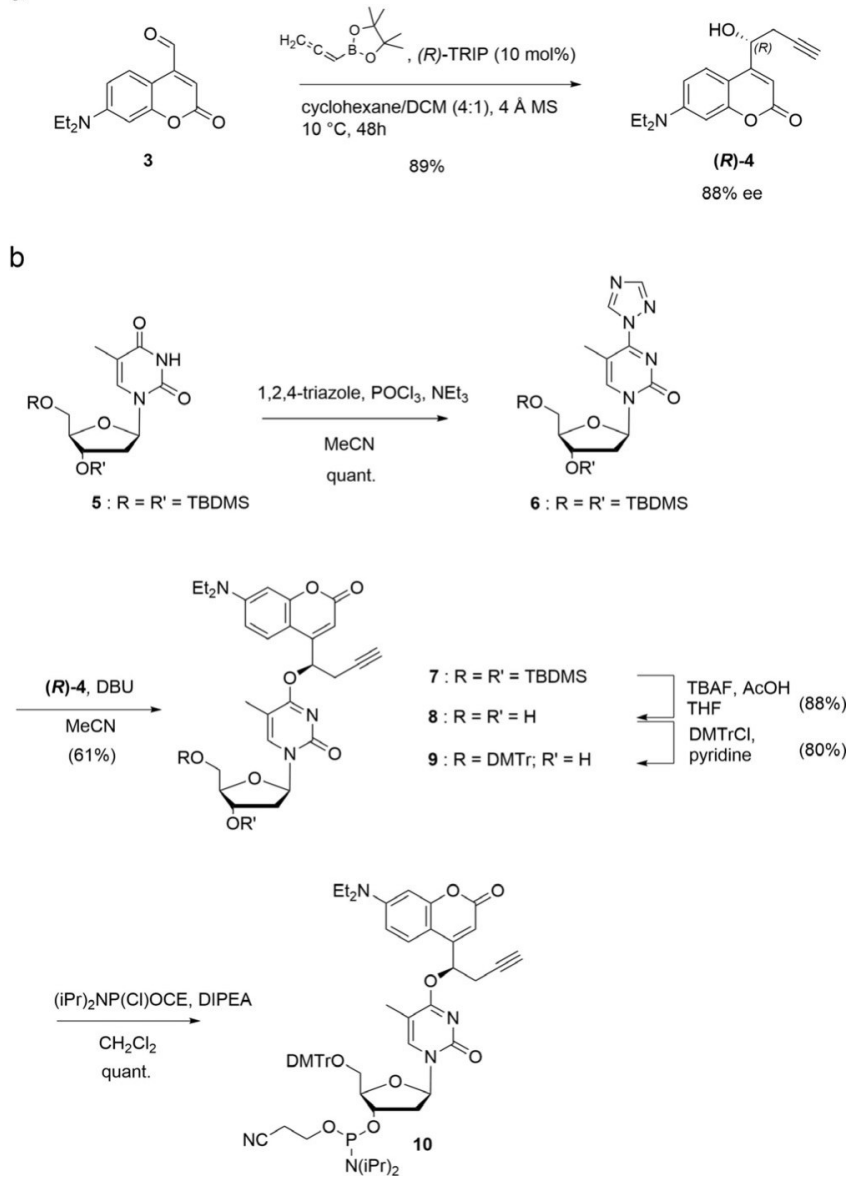

Scheme 1. Enantioselective synthesis of 7-(diethylamino)-4-(1-hydroxybut-3yn-1-yl)coumarin- (DEACBY-OH). (a) Synthesis of a diastereomeric pure DEACBY-caged thymidine for the solid phase synthesis of caged DNA $\mathbf{2} \mathbf{a}-\mathbf{i}$. (b) Incorporation into protected phosphoramidite $\mathbf{1 0}$ for DNA solid phase synthesis.

eluted fractions of the DEACBY screening are in every case the $(S)$ - and the later eluted the (R)-configured derivatives (Figures 6 and S9 in the Supporting Information). In the next step we measured melting temperatures of all $(R)-\mathbf{2} \mathbf{b}-\mathbf{i}$ and $(S)-\mathbf{2} \mathbf{b}-\mathbf{i}$ derivatives (Figure 3, circles and rhombi). The first important result of this screening is that in all cases with oligonucleotides $\mathbf{2} \mathbf{b}$-i the early eluted (S)-diastereomers exhibit considerably lower melting temperatures compared to the $(R)$-diastereomers. This observation is in line with a previous study in which the $(S)$-configured NPE-caged cytosine exhibited a pronounced lower duplex stability than the $(R)$-configured derivative. ${ }^{[43]}$ The second important conclusion is that the most efficient design for single nucleobase caging in our experiments turns out to be (S)-configured diphenylmethyltriazole-coumarin (DPMTC) in (S)-2 $\mathrm{f}$ with a melting temperature of only $33.1^{\circ} \mathrm{C}\left(\Delta T_{\mathrm{m}}=\right.$ $15.8^{\circ} \mathrm{C}$ compared to the native DNA with only one single modified residue!). The lowest melting temperature previously achieved for this DNA sequence was $35.4^{\circ} \mathrm{C}\left(\Delta T_{m}=13.5^{\circ} \mathrm{C}\right)$ in a study, in which the achiral 7-diethylaminocoumarin (DEACM) cage was attached directly at $\mathrm{N}^{3}$ position of 2'-deoxythymidine $\left(\mathrm{dT}^{\mathrm{NDEACM}}\right){ }^{[44]}$
However, this design, using DEACM, was not suitable for photo-uncaging reactions of nucleobases, since $\mathrm{C}-\mathrm{N}$-bond cleavage with light is extremely inefficient. As the oligonucleotide modified with bulky cage (S)- $\mathbf{2} \mathbf{f}$ revealed the lowest melting temperature, we investigated also the uncaging properties of DPMTC caged DNA at $405 \mathrm{~nm}$ irradiation for this promising cage employing ferrioxalate actinometry (Figure 8) for both, the $(S)$ - and $(R)$-diastereomer (Table 1). The uncaging efficiencies, defined by the product of molar extinction coefficient and uncaging quantum yield $(\varepsilon \cdot \phi)$, are found to be in the same

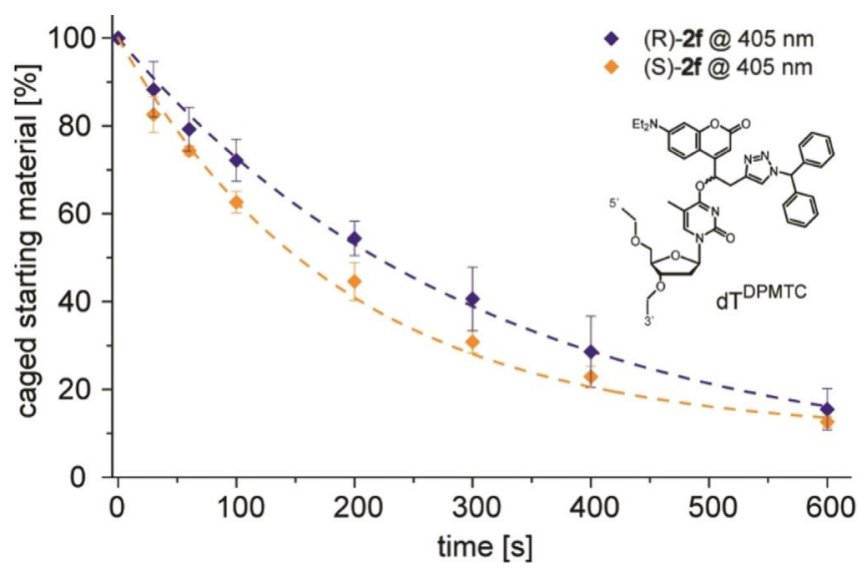

Figure 8. Uncaging kinetics of diphenylmethyltriazole-coumarin- (DPMTC) caged oligonucleotides $(R)$ - and (S)-2 $\mathrm{f}$ at $405 \mathrm{~nm}$ irradiation $(220 \mathrm{pmol}$, $16.9 \mu \mathrm{M}, 47 \mathrm{~mW})$.

Table 1. Photochemical properties of the bulky DPMTC-caged oligonucleotide $(R)$ - and (S)-2 $\mathbf{f}$ diastereomers (measured in PBS buffer at $\mathrm{pH}$ 7.4).

\begin{tabular}{|lllll|} 
& $\lambda[\mathrm{nm}]$ & $\phi$ & $\varepsilon\left[\mathrm{M}^{-1} \mathrm{~cm}^{-1}\right]^{]}$ & $\varepsilon \cdot \phi\left[\mathrm{M}^{-1} \mathrm{~cm}^{-1}\right]$ \\
\hline$(R)-\mathbf{2} \mathbf{f}$ & 405 & 0.007 & 22950 & 170 \\
$(S)-\mathbf{f}$ & 405 & 0.011 & 23800 & 258 \\
\hline
\end{tabular}

range as previously reported for the $\mathrm{O}^{4}$-caged $\mathrm{dT}^{\text {DEACM }}$ $\left(\varepsilon(405) \cdot \phi=247 \mathrm{M}^{-1} \mathrm{~cm}^{-1}\right)$. Uncaging of $(S)-2 \mathrm{f}$ proceeded significantly faster (with a 1.5-fold higher uncaging efficiency compared to $(R)-\mathbf{2} f$ ). Noteworthy, the $(S)$-configured oligonucleotide $\mathbf{2} \mathbf{f}$ not only features the lowest melting temperature, but also better uncaging properties.

\section{Investigation of caged DNA conformations by molecular dynamics simulations}

The NPBY and DEACBY screenings (Figure 7) reveal a melting temperature plateau where-within the error-the destabilization of the duplex depends only weakly on the specific bulky triazole residues. To understand how our caged DNA design interferes with the native duplex we proceeded with molecular dynamics (MD) simulations of the native DNA, $\mathrm{dT}^{\text {DPMTC}}$-caged $(S)$ - or $(R)-\mathbf{2} \mathbf{f}$ and $\mathrm{dT}^{\text {DEACM }}$-caged DNA variants. These caged nucleobases are sterically more demanding than the $\mathrm{dC}^{\mathrm{NPPM}}$ cages discussed above in the NMR to MD structure comparison, for 


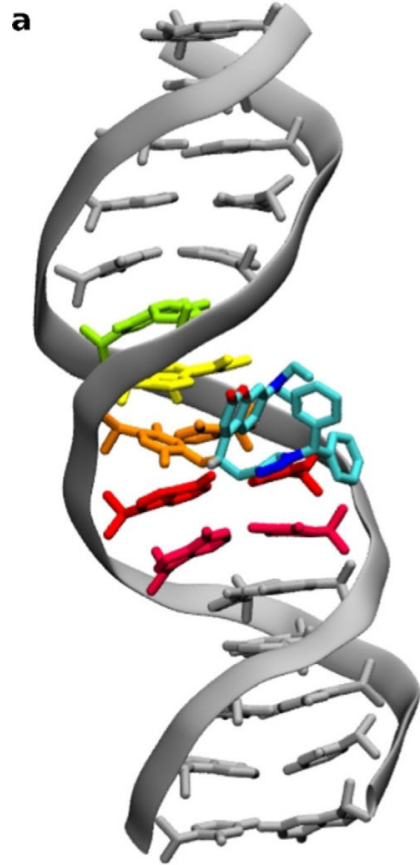

d

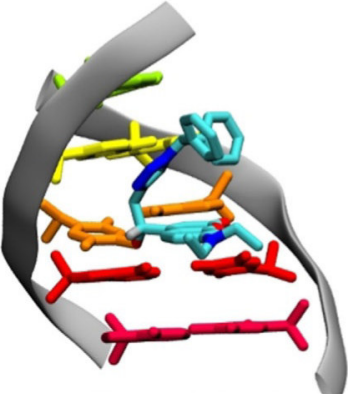

(R)-DPMTC b

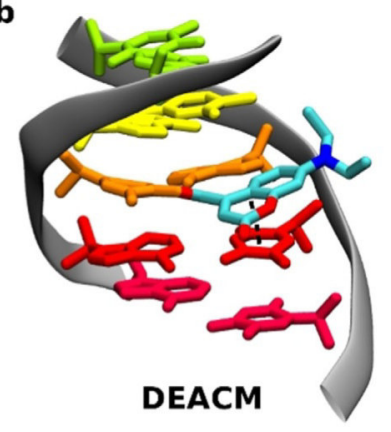

C

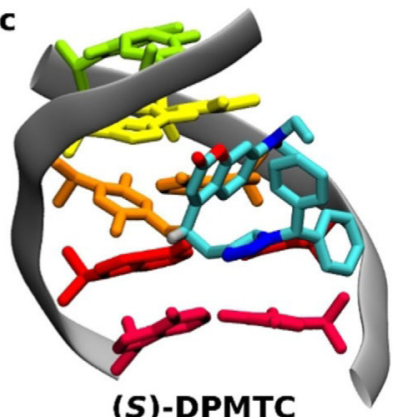

e

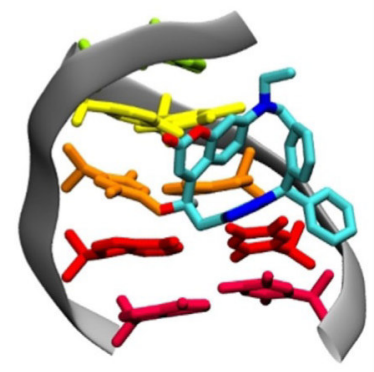

(R)-DPMTC

Figure 9. Conformation of caged DNA in MD simulations. (a) The caged DNA keeps its helical structure as shown for DPMTC-caged DNA (S)-2 f. Highlighted base pairs are A6·T25 (lime-green), A7·T24 (yellow), X8.A23 (orange), A9.T22 (red) and A10.T21 (purple-red). Representative structures of DEACMcaged DNA (b) and DPMTC-caged DNA (S)-2 $\mathbf{f}(\mathrm{c})$ with focus on the caged thymidine T8. (d) shows $(R)-2 \mathrm{f}$ with an initially $180^{\circ}$ rotated cage in the major groove, which rotates back into the favored conformation within simulation time (e). Hydrogens are not shown except at the stereogenic center.

which we could directly validate structures from MD by NMR. Computational predictions have already previously proven to be helpful in the design of caged oligonucleotides. For example, Tang et al. investigated caging of the backbone phosphates of siRNA. ${ }^{[48]}$

The stability of the caged DNA was investigated in five independent simulations per cage (cumulative $1 \mu \mathrm{s} /$ cage). Overall, the DNA retained its helical structure in all simulations (Figure 9 a). We obtained stable simulations with heavy atom position RMSDs to the average structure for $\mathrm{dT}^{\text {DEACM }}, \mathrm{dT}^{(5) \text {-DPMTC }}$ and $\mathrm{dT}^{(R) \text {-DPMTC }}$ of $2.1 \pm 0.7 \AA, 1.7 \pm 0.5 \AA$ and $1.7 \pm 0.4 \AA$ (see Supporting Information). Even for sterically challenging cages, the modified bases still fit into the double helix, aided by the flexibility of the bonds connecting the cages with the aromatic ring of thymine. These results are in agreement with previous

NMR investigations. ${ }^{[43]}$ They are also consistent with our results for the NPPM cages, which show that the cages do not adopt a fully solvent accessible conformation.

However, the caged bases were found to be more flexible in the simulations (see Supporting Information). Due to the attachment of the cage at the thymidine $\mathrm{O}^{4}$ position, the dT base has no hydrogen donor at the $\mathrm{N}^{3}$ position. The native hydrogen bond between $\mathrm{dT} \mathrm{N}^{3}$ and opposite $\mathrm{dA} \mathrm{N} \mathrm{N}^{1}$ cannot be formed. The only remaining hydrogen bond between the caged thymidine and opposite adenine is a non-Watson-Crick hydrogen bond, namely between $\mathrm{dT} \mathrm{O}^{2}$ and $\mathrm{dA} \mathrm{N}^{6}$. This enhances the flexibility of the caged bases and allows them to shear in the base plane. Importantly, bulky substituents are in general pointing out in the major groove or clash with a neighboring base. With the achiral DEACM attached to thymidine, the caging group points along the major groove (Figure $9 \mathrm{~b}$ ). The aromatic DEACM forms a putative sandwich $\pi$ - interaction with thymidine in the complementary strand (T22, red in Figure $9 \mathrm{~b}$ ). Simulations of the bulky ( $R$ )- and (S)-DPMTC derivatives reveal that the coumarin group points toward the preceding residue (A7, yellow in Figure 9c), forming a T-shape, likely stabilized by $\pi$-stacking interactions. ${ }^{[4]}$ In contrast, the larger triazole substituent occupies the major groove (Figure 9c). While DEACM is able to rotate within the major groove, $(R)$-DPMTC and $(S)$ DPMTC cages seem to fit rigidly into the local helix structure. For $(R)$ - DPMTC we find rotations of the diphenyl group around the triazole. (S)-DPMTC is even less flexible, only the phenyl groups are free to rotate. To rule out any bias from the starting geometry, we performed an additional simulation for (R)-DPMTC, with the cage initially rotated by $180^{\circ}$ along the base plane, so that the position of the smaller coumarin and larger triazole substituents were interchanged (Figure 9d). After 150 ns of simulation time the cage rotated back, with the smaller substituent facing the A7 base, while the larger triazole substituent again points into the major groove (Figure $9 \mathrm{e}$ ).

With the structural models at hand, it is tempting to speculate about the origin of the duplex destabilization of our different cages. Bulky substituents point into the major groove, while smaller residues pack close to the base pairs. For DEACM, $\pi$-stacking interactions of the cage with bases of the complementary strand may even contribute to a stabilization of the DNA duplex. In contrast, $\alpha$-substituted bulky cages such as DPMTC disturb the local duplex structure and additionally expose large, hydrophobic groups to the polar solvent. More favorable interactions between DNA and cages in the singlestranded state compared to the duplex state would also contribute to duplex destabilization. For instance, hydrophobic groups exposed to the solvent such as the diphenyl group of DPMTC may be stacked to the bases in the single-stranded state, which would decrease the penalty for exposing a nonpolar group.

Finally, we hypothesize that for the bulky caged DNAs the individual residues at the triazole do not affect the duplex stability significantly, as long as they exceed the bulkiness of the cage moiety and, as shown by the simulations, point into the major groove. This hypothesis agrees with the observed plateau of melting temperatures in our click screenings. 


\section{Conclusions}

Optimization of the temporary, local destabilization of oligonucleotide hybridization with photocages is important since numerous examples have shown that it is not necessary to completely block duplex association in order to have a biological effect. ${ }^{[21,50,51]}$

In summary, we have followed two alternative strategies to maximize the destabilization efficiency of incorporating a single photocaged group into duplex DNA. Such optimization is vital to extend the applicability of photocaged DNA for in vivo studies. In a first series, we added a bulky group directly in the $\alpha$-position of the cage moiety, introducing the phenyl (NPPM) and the adamantyl group (NPAM) as bulky substituents. By NMR, we assigned the signals of each of the diastereomeric compounds, determined the structure around the modified site and characterized the extent of local destabilization of the DNA duplex, showing that the two adjacent base-paired nucleotides are destabilized at both, $5^{\prime}$ and $3^{\prime}$ site of the duplex. The difference $\Delta \Delta T_{\mathrm{m}}$ in destabilization of the two diastereomeric caged nucleotides becomes smaller with increasing steric demand of the substituent. In this part of the study, we established that the results of NMR spectroscopy and MD simulations agree well.

In a second series, we then devised a medium throughput strategy to screen a large number of different photolabile groups and bulky substituents using a post-synthetic modification by $\mathrm{Cu}$-catalyzed azide-alkyne cycloaddition click reaction. We show that diphenylmethyltriazole-coumarin (DPMTC) connected in (S)-configuration to the nucleobase exhibits the largest duplex destabilization $\left(\Delta T_{\mathrm{m}}=15.8^{\circ} \mathrm{C}\right)$ ever measured for a single base-caged DNA. With DPMTC, we thus provide a powerful new coumarin cage, which can be applied to efficient light regulation of functional oligonucleotides at wavelengths over $400 \mathrm{~nm}$. Moreover, due to the modular character of click reactions this strategy will be easily applicable to more redshifted photolabile protecting groups that are currently under investigation. We have screened an array of click partners to understand the mechanism of duplex destabilization. This opens up the possibility to study also moieties with interesting biological activities.

With the aid of molecular dynamic simulations, we investigated, how bulky cages influence the duplex stability at atomic resolution. We hypothesize that the triazole residues, as they are sterically more demanding, point into the major groove. By contrast, the coumarin cage moiety is tolerated close to the neighboring base-pair forming a T-shape structure. A detailed thermodynamic and kinetic characterization of the association and dissociation process will help to understand the effect of the bulky cages entirely. Those experiments are currently in progress.

\section{Acknowledgements}

M.H. and L.S.S. thank Dr. J. Köfinger for insightful discussions and $\mathrm{M}$. Vögele for help with the cage parameterization. This re- search was supported by the Deutsche Forschungsgemeinschaft (GRK 1986 "CLiC"), the Fonds der Chemischen Industrie (fellowship to P.S.) and the European commission (EUROPOL to G.P.). M.H, L.S.S and G.H. acknowledge financial support by the German Research Foundation (CRC 902: Molecular Principles of RNA Based Regulation) and the Max Planck Society. H.S., A.H. and G.H. are member of the DFG-funded cluster of excellence Macromolecular Complexes (EXC115). Work at BMRZ is supported by the state of Hesse.

\section{Conflict of interest}

The authors declare no conflict of interest.

Keywords: click chemistry - computational chemistry • DNA NMR spectroscopy • nucleobases • oligonucleotides photochemistry

[1] N. Ankenbruck, T. Courtney, Y. Naro, A. Deiters, Angew. Chem. Int. Ed. 2018, 57, 2768-2798; Angew. Chem. 2018, 130, 2816-2848.

[2] M. J. Hansen, W. A. Velema, M. M. Lerch, W. Szymanski, B. L. Feringa, Chem. Soc. Rev. 2015, 44, 3358-3377.

[3] G. Bort, T. Gallavardin, D. Ogden, P. I. Dalko, Angew. Chem. Int. Ed. 2013 52, 4526-4537; Angew. Chem. 2013, 125, 4622-4634.

[4] P. Klán, T. Šolomek, C. G. Bochet, A. Blanc, R. Givens, M. Rubina, V. Popik, A. Kostikov, J. Wirz, Chem. Rev. 2013, 113, 119-191.

[5] X. Ai, J. Mu, B. Xing, Theranostics 2016, 6, 2439-2457.

[6] H. K. Agarwal, R. Janicek, S.-H. Chi, J. W. Perry, E. Niggli, G. C. R. EllisDavies, J. Am. Chem. Soc. 2016, 138, 3687-3693.

[7] G. C. R. Ellis-Davies, Nat. Methods 2007, 4, 619-628.

[8] M. M. Mahmoodi, D. Abate-Pella, T. J. Pundsack, C. C. Palsuledesai, P. C. Goff, D. A. Blank, M. D. Distefano, J. Am. Chem. Soc. 2016, 138, 58485859

[9] A. Gautier, C. Gauron, M. Volovitch, D. Bensimon, L. Jullien, S. Vriz, Nat. Chem. Biol. 2014, 10, 533-541.

[10] J. Hemphill, E. K. Borchardt, K. Brown, A. Asokan, A. Deiters, J. Am. Chem. Soc. 2015, 137, 5642-5645.

[11] A. S. Baker, A. Deiters, ACS Chem. Biol. 2014, 9, 1398-1407.

[12] J. Buck, B. Fürtig, J. Noeske, J. Wöhnert, H. Schwalbe, Proc. Natl. Acad. Sci. USA 2007, 104, 15699-15704.

[13] F. Buhr, J. Kohl-Landgraf, S. tom Dieck, C. Hanus, D. Chatterjee, A. Hegelein, E. M. Schuman, J. Wachtveitl, H. Schwalbe, Angew. Chem. Int. Ed. 2015, 54, 3717-3721; Angew. Chem. 2015, 127, 3788-3792.

[14] C. Brieke, F. Rohrbach, A. Gottschalk, G. Mayer, A. Heckel, Angew. Chem. Int. Ed. 2012, 51, 8446-8476; Angew. Chem. 2012, 124, 8572-8604.

[15] B. K. Ruble, S. B. Yeldell, I. J. Dmochowski, J. Inorg. Biochem. 2015, 150, $182-188$.

[16] P. Wenter, B. Fürtig, A. Hainard, H. Schwalbe, S. Pitsch, Angew. Chem. Int. Ed. 2005, 44, 2600-2603; Angew. Chem. 2005, 117, 2656-2659.

[17] P. K. Jain, V. Ramanan, A. G. Schepers, N. S. Dalvie, A. Panda, H. E. Fleming, S. N. Bhatia, Angew. Chem. Int. Ed. 2016, 55, 12440-12444; Angew. Chem. 2016, 128, 12628-12632.

[18] Y. Ji, J. Yang, L. Wu, L. Yu, X. Tang, Angew. Chem. Int. Ed. 2016, 55, 2152 2156; Angew. Chem. 2016, 128, 2192-2196.

[19] C. T. Huynh, M. K. Nguyen, G. Y. Tonga, L. Longé, V. M. Rotello, E. Alsberg, Adv. Healthcare Mater. 2016, 5, 305-310.

[20] F. Schäfer, J. Wagner, A. Knau, S. Dimmeler, A. Heckel, Angew. Chem. Int. Ed. 2013, 52, 13558-13561; Angew. Chem. 2013, 125, $13801-13805$.

[21] A. Heckel, M. C. R. Buff, M. L. Raddatz, J. Müller, B. Pötzsch, G. Mayer, Angew. Chem. Int. Ed. 2006, 45, 6748-6750; Angew. Chem. 2006, 118, $6900-6902$.

[22] D. Lovatt, B. K. Ruble, J. Lee, H. Dueck, T. K. Kim, S. Fisher, C. Francis, J. M. Spaethling, J. A. Wolf, M. S. Grady, A. V. Ulyanova, S. B. Yeldell, J. C. Griepenburg, P. T. Buckley, J. Kim, J.-Y. Sul, I. J. Dmochowski, J. Eberwine, Nat. Methods 2014, 11, 190-196. 
[23] D. Zhang, C. Y. Zhou, K. N. Busby, S. C. Alexander, N. K. Devaraj, Angew. Chem. Int. Ed. 2018, 57, 2822-2826.

[24] C. Helmling, D.-P. Klötzner, F. Sochor, R. A. Mooney, A. Wacker, R. Landick, B. Fürtig, A. Heckel, H. Schwalbe, Nat. Commun. 2018, 9, 944.

[25] D. D. Young, M. O. Lively, A. Deiters, J. Am. Chem. Soc. 2010, 132, $6183-$ 6193.

[26] J. M. Govan, D. D. Young, H. Lusic, Q. Liu, M. O. Lively, A. Deiters, Nucleic Acids Res. 2013, 41, 10518-10528.

[27] T. Lucas, F. Schäfer, P. Müller, S. A. Eming, A. Heckel, S. Dimmeler, Nat Commun. 2017, 8, 15162.

[28] L. Zhang, D. Liang, Y. Wang, D. Li, J. Zhang, L. Wu, M. Feng, F. Yi, L. Xu, L. Lei, Q. Du, X. Tang, Chem. Sci. 2018, 9, 44-51.

[29] X. Tang, J. Swaminathan, A. M. Gewirtz, I. J. Dmochowski, Nucleic Acids Res. 2007, 36, 559-569.

[30] P. Seyfried, L. Eiden, N. Grebenovsky, G. Mayer, A. Heckel, Angew. Chem. Int. Ed. 2017, 56, 359-363; Angew. Chem. 2017, 129, 365-369.

[31] S. Yamazoe, I. A. Shestopalov, E. Provost, S. D. Leach, J. K. Chen, Angew. Chem. Int. Ed. 2012, 51, 6908-6911; Angew. Chem. 2012, 124, $7014-$ 7017.

[32] J. C. Griepenburg, T. L. Rapp, P. J. Carroll, J. Eberwine, I. J. Dmochowski, Chem. Sci. 2015, 6, 2342-2346.

[33] S. Takamori, S. Yamaguchi, N. Ohashi, T. Nagamune, Chem. Commun. 2013, 49, 3013

[34] S. Yamaguchi, Y. Chen, S. Nakajima, T. Furuta, T. Nagamune, Chem. Commun. 2010, 46, 2244-2246.

[35] M. T. Richers, J. M. Amatrudo, J.P. Olson, G. C. R. Ellis-Davies, Angew. Chem. Int. Ed. 2017, 56, 193-197; Angew. Chem. 2017, 129, 199-203.

[36] I. Ivani, P. D. Dans, A. Noy, A. Pérez, I. Faustino, A. Hospital, J. Walther, P. Andrio, R. Goñi, A. Balaceanu, G. Portella, F. Battistini, J. L. Gelpí, C. González, M. Vendruscolo, C. A. Laughton, S. A. Harris, D. A. Case, M. Orozco Nat. Methods 2016, 13, 55-58.

[37] R. Galindo-Murillo, J. C. Robertson, M. Zgarbová, J. Šponer, M. Otyepka, P. Jurečka, T. E. Cheatham III, J. Chem. Theory Comput. 2016, 12, 4114 4127.
[38] M. Krepl, M. Zgarbová, P. Stadlbauer, M. Otyepka, P. Banáš, J. Koča, T. E. Cheatham III, P. Jurečka, J. Šponer, J. Chem. Theory Comput. 2012, 8, $2506-2520$.

[39] M. Zgarbová, F. J. Luque, J. Šponer, T. E. Cheatham III, M. Otyepka, P. Jurečka, J. Chem. Theory Comput. 2013, 9, 2339-2354.

[40] M. Zgarbová, J. Šponer, M. Otyepka, T. E. Cheatham III, R. Galindo-MurilIo, P. Jurečka, J. Chem. Theory Comput. 2015, 11, 5723-5736.

[41] P. D. Dans, I. Ivani, A. Hospital, G. Portella, C. González, M. Orozco, Nucleic Acids Res. 2017, 45, 4217-4230.

[42] L. S. Stelzl, N. Erlenbach, M. Heinz, T. F. Prisner, G. Hummer, J. Am. Chem. Soc. 2017, 139, 11674-11677.

[43] H. S. Steinert, F. Schäfer, H. R. A. Jonker, A. Heckel, H. Schwalbe, Angew. Chem. Int. Ed. 2014, 53, 1072-1075; Angew. Chem. 2014, 126, $1090-$ 1093.

[44] A. Rodrigues-Correia, D. Knapp-Bühle, J. W. Engels, A. Heckel, Org. Lett. 2014, 16, 5128-5131.

[45] F. Schäfer, K. B. Joshi, M. A. H. Fichte, T. Mack, J. Wachtveitl, A. Heckel, Org. Lett. 2011, 13, 1450-1453.

[46] L. R. Reddy, Org. Lett. 2012, 14, 1142-1145.

[47] P. Jain, H. Wang, K. N. Houk, J. C. Antilla, Angew. Chem. Int. Ed. 2012, 51 1391 -1394; Angew. Chem. 2012, 124, 1420-1423.

[48] L. Wu, F. Pei, J. Zhang, J. Wu, M. Feng, Y. Wang, H. Jin, L. Zhang, X. Tang, Chem. Eur. J. 2014, 20, 12114-12122.

[49] M. O. Sinnokrot, C. D. Sherrill, J. Phys. Chem. A 2004, 108, 10200-10207.

[50] C. Helmling, D.-P- Klötzner, F. Sochor, R. A. Mooney, A. Wacker, R. Landick, B. Fürtig, A. Heckel, H. Schwalbe, Nat. Commun. 2018, 9, 944-952.

[51] V. Mikat, A. Heckel, RNA 2007, 13, $2341-2347$.

Manuscript received: August 7, 2018

Accepted manuscript online: September 10, 2018

Version of record online: November 5, 2018 